\title{
Similarity between craze morphology and shear-band morphology in polystyrene
}

\author{
T. E. BRADY*, G. S. Y. YEH \\ Department of Materials and Metallurgical Engineering and Macromolecular Research Center, \\ University of Michigan, Ann Arbor, Michigan, USA
}

The formation of shear bands and crazes in thin films as well as in bulk samples of polystyrene were examined in the electron microscope using a variety of replication techniques. The morphologies of shear bands and crazes are quite similar both depending initially upon the relative shear displacement of 400 to $1000 \AA$ domains. As deformation continues and orientation increases, fibrils varying from 50 to $700 \AA$ are formed within the deformation zone, lateral constraint of the normal Poisson contraction causing voids to form in the crazes but not in the shear bands. Shear-band width was found not to be a unique function of either temperature or strain-rate and both craze and shear-band morphologies were found not to be strong functions of molecular weight. Regardless of molecular weight, fibrils formed within the deformation zone were always on the order of a few hundred Angstroms in diameter. However, for thin films of molecular weight less than 20000 insufficient numbers of tie molecules between fundamental structural units or domains made it difficult for these fibres to span the craze width.

\section{Introduction}

A wealth of technical literature and several comprehensive reviews [1-3] are devoted to the phenomenon of "crazing" in glassy amorphous polymers, but there are few studies which consider a very closely related phenomenon, "shear banding", in detail. In this investigation we have employed the electron microscope to examine both crazes and shear bands in polystyrene, and found that their basic morphologies are quite similar.

Crazing and shear banding are both modes of plastic deformation, but crazes usually occur in response to a tensile stress under a high lateral constraint which inhibits the normal Poisson contraction from accompanying elongation of the polymer [2]. In many polymer systems, this constraint creates a fibrillar morphology consisting of highly oriented 100 to $300 \AA$ fibrils interspaced by voids of the same dimensions (1-11], very much like an "open-cell foam", according to Kambour and Holick [8].

The transition between the undeformed polymer matrix and the craze itself is quite sharp $[8,9]$ and the fibrils have a characteristic, and nearly constant, diameter $[4,8]$. Within polystyrene crazes, Beahan et al [4] have observed a discrete 75 to $150 \AA$ beaded structure along the fibrils which, they suggest, is evidence for a domain structure in polystyrene. However, it is not yet clear to what extent these 75 to $150 \AA$ domains are related to the much smaller 15 to $45 \AA$ ordered domains which Yeh [12] identified using dark-field electron microscopy.

In contrast to crazing, the formation of a shear band is controlled by shear stresses which actually encourage lateral movement and hence prevent the void formation which is associated with crazing. Although shear banding has also been observed in many polymer systems [13-18], there have been few attempts to characterize the morphology of this type of plastic deformation zone. Argon et al [16] have reported that shear zones in polystyrene consist of many individual 0.5 to $1.0 \mu \mathrm{m}$ wide shear bands, each supporting a shear strain of from 1.0 to 2.2 . In the same study, it was reported that the shear deformation within these bands is completely recoverable at temperatures above $T_{\mathrm{g}}$ and also that the optic axis inside the bands is consistent with the

*Present address: Owens-Illinois, Toledo, Ohio

(C) 1973 Chapman and Hall Ltd. 
anticipated molecular orientation calculable from the magnitude of the observed shear strains [16]. Electron micrographs published by Argon et al [16] and Brady and Yeh [13] demonstrate that, as in the case of crazing, the boundary between the band and the polymer matrix is quite sharp. Bowden and Raha [14] published a micrograph of a thin section taken through a polystyrene shear band in which there was no evidence of a discrete microstructure, but Brady and Yeh have reported surface replica studies which indicate the presence of a 100 to $1000 \AA$ fibrillar microstructure within the polystyrene shear bands.

Several recent studies have suggested that crazing and shear banding are somehow related. It is generally held that crazes will not form in either compression or in pure shear, but Harris and Ward [6] have observed the usual crazing normal to the tensile stress as well as what appear to be crazes along the shear direction in oriented polyethylene terephthalate. In another study, Haward et al [19] have compared the comprehensive yield behaviour to polystyrene with tensile crazing and have found that the strain-rate dependencies of the tensile crazing stress and the compressive yield stress respond to temperature in the same way, and have suggested therefore, that crazing and yielding may involve similar molecular motions.

If, as the above studies suggest, there is a close relationship between molecular processes involved in crazing and shear banding, and if crazing can occur in pure shear as well as in tension, then it is reasonable to expect the microstructures resulting from these two modes of deformation to be quite similar. In this investigation, we have pursued this possibility by carefully examining and comparing the microstructures of crazes and shear bands in both thin films and bulk samples of polystyrene.

In order to understand what contributes most to the integrity of the deformed polymer, we have also examined these deformation modes as functions of molecular weight. Previous studies of polystyrene have shown that time-to-failure under load [20] as well as the number of crazes per unit volume [21] both increase with increasing molecular weight, suggesting that the number of tie molecules may be a critical parameter. The study on the effect of molecular weight on craze morphology has also been recently taken up by others [22, 23] and their preliminary results appear to agree with ours.

\section{Experimental}

Shell general-purpose atactic polystyrene (GPAPS) (mol wt 250000) and Dow EP-1340-128 isotactic polystyrene ( $\mathrm{mol}$ wt 550000 ) were used in this investigation. In addition, a series of narrow distribution molecular weight atactic polystyrenes $(4800,15000,19000,51000,113000$ 265000,402000 ) was also obtained from either the Dow Chemical Company or the Pressure Chemical Company and used to study the effects of molecular weight.

Thin films of atactic polystyrene (APS) and isotactic polystyrene (IPS) were cast on glass slides from $0.7 \%$ benzene solutions. These films ( $\sim 3000$ to $5000 \AA$ thick) were floated onto the surface of distilled water and were picked up on 0.003 in.) Mylar strips. Drawing was achieved by first heating above $100^{\circ} \mathrm{C}$ for $10 \mathrm{~min}$ (both to rid the film of most excess solvent and to increase coherency between film and substrate), and then uniformly drawing the substrate to specific elongations in a hand stretcher. Surface replicas of the deformed thin films were prepared using conventional platinum-carbon shadowing techniques.

Both crazes and shear bands were also formed in bulk polystyrene compression samples and their morphologies were examined using similar replication techniques.

While craze morphology was observed only by direct replication of the deformation zone, shear band morphology was studied as well by (1) etching with chromic-sulphuric acid, (2) applying a thin layer of platinum prior to deformation and replication, and (3) replication of an artificially created internal compression surface. Details of these techniques are included where appropriate.

\section{Results}

\subsection{Thin films}

Figs. $1 a$ and $b$ are reproduced from [13] and contrast the surface textures of quenched and annealed thin films of GP-APS drawn $25 \%$ at room temperature on a Mylar substrate. By drawing to several elongations less than $25 \%$, we determine that crazes were the first deformation zones to form. Crazing was followed by shear yielding and cold flow, clearly evident in Fig. 1 as discrete shear lines.

The crazes consist of fibrillar material $(\sim 300$ $\AA$ in width) interspaced by voids of similar dimensions (see insert, Fig. 1b). Both Kambour and Robertson [2] and Beahan et al have 

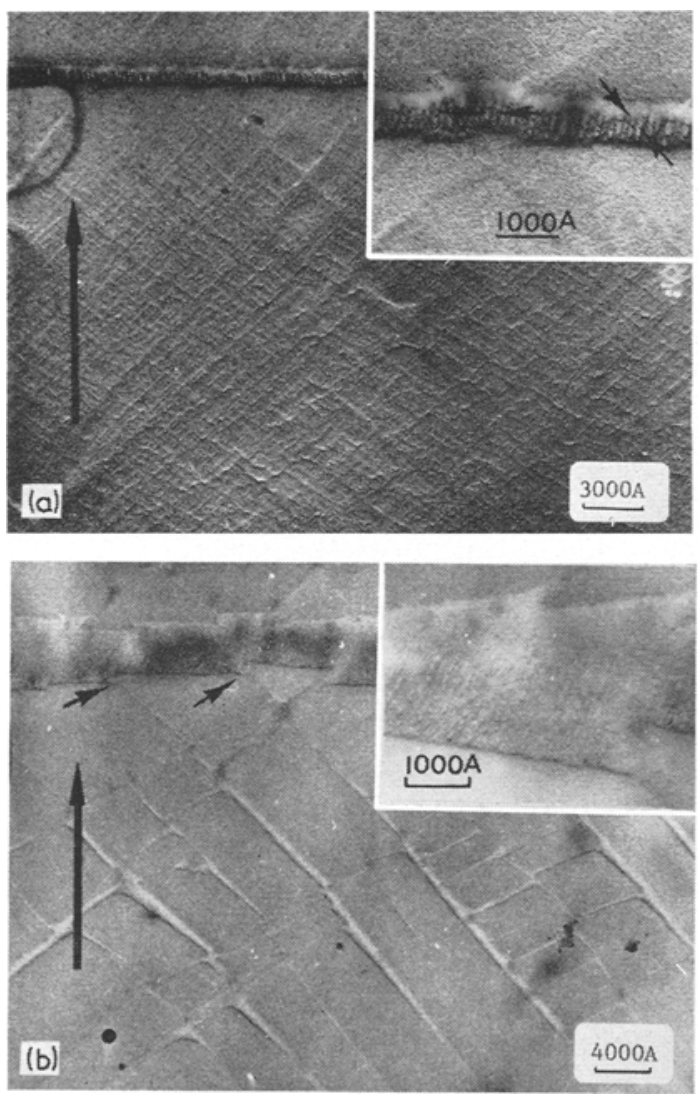

Figure I (a) Pt-C surface replica of APS thin film quenched from $105^{\circ} \mathrm{C}$ and drawn $25 \%$ on a Mylar substrate. The vertical arrow indicates the draw direction and the small arrows show 200 to $300 \AA$ fibrils within a craze [12]. (b) Pt-C surface replica of an APS thin film annealed for 3 days at $75^{\circ} \mathrm{C}$ and $25 \%$ on a Mylar substrate. The vertical arrow indicates the draw direction and the small arrows show steps which form when shear bands reach the edge of a craze [12].

reported a similar morphology for polystyrene crazes.

Annealing below $T_{\mathrm{g}}$ promotes even more extreme localized deformation. This is demonstrated in Fig. 1b where fewer, but much wider, shear bands are evident. Fig. 1a, however, demonstrates that even quenched APS thin films deform by localized shear banding. When a shear band reaches the edge of a craze, a step forms, emphasizing the localized character of the shear band (see Fig. 1b).

Similar results were found for partially crystalline IPS thin films as shown in Figs. $2 a$ and $b$. Other important features of the deformation process are also demonstrated in Fig. 2. Fig. 2a
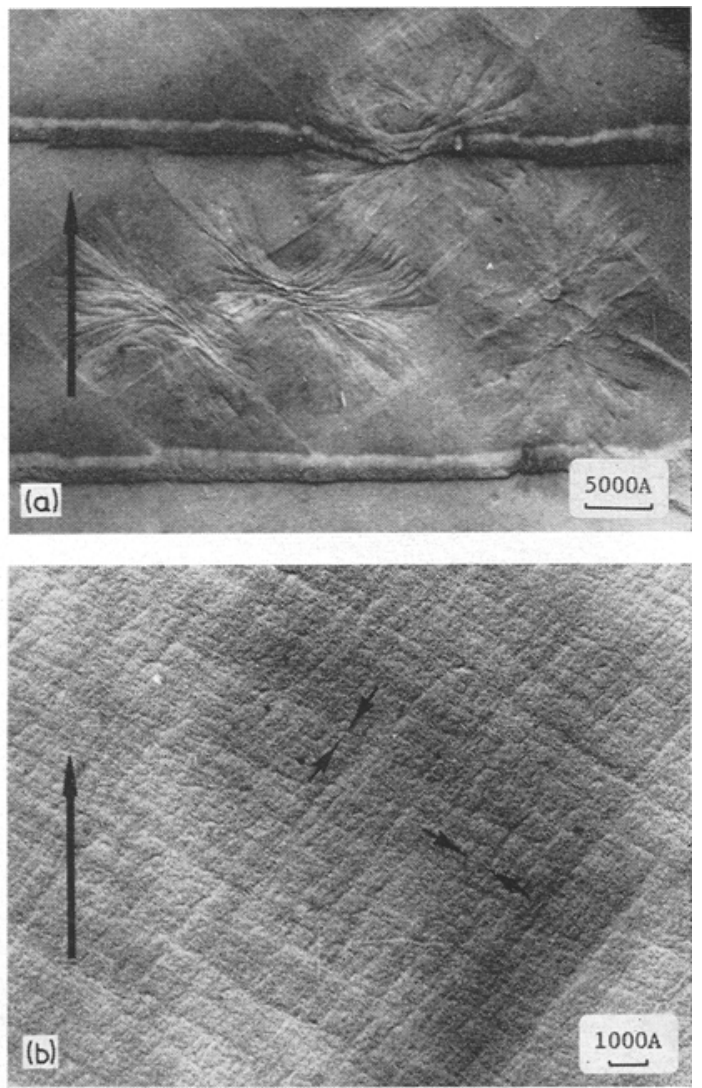

Figure 2 (a) Pt-C surface replica of a partially crystalline IPS thin film drawn $25 \%$ on a Mylar substrate. The vertical arrow indicates the draw direction. (b) A higher magnification of Fig. 2a with small arrows showing interspacings of 200 and $500 \AA$. The vertical arrow indicates the draw directior.

shows that the crystalline spherulites deform only after substantial deformation has occurred in the connecting amorphous regions. Fig. $2 \mathrm{~b}$ demonstrates that shear line interspacings are consistently between 200 to $700 \AA$. Also, in agreement with previous investigators $[11,16]$, we find that the crazes, shear bands and steps shown in the above micrographs are all recoverable above $T_{\mathrm{g}}$.

To study further the deformation of the lamellae within the IPS spherulites, semi-crystalline films were prepared by allowing a $0.7 \%$ benzene solution of IPS to flow rapidly along a glass slide as the benzene evaporated. Residual molecular orientation along the flow direction resulted in formation of crystalline lamellae oriented perpendicular to the flow direction when the film. was crystallized for $15 \mathrm{~min}$ at $170^{\circ} \mathrm{C}$. This 
oriented lamellar morphology provided a convenient means for separating and deforming the individual crystalline lamellae. When these semicrystalline films were drawn $25 \%$ on a Mylar substrate (in a direction perpendicular to the lamellar orientation), crazing and shear banding were again the primary modes of deformation. However, as the crazes propagated through the crystalline regions, the lamellae separated and appeared to break up into 100 to $200 \AA$ domains. This is shown in Fig. $3 b$ where the small arrows
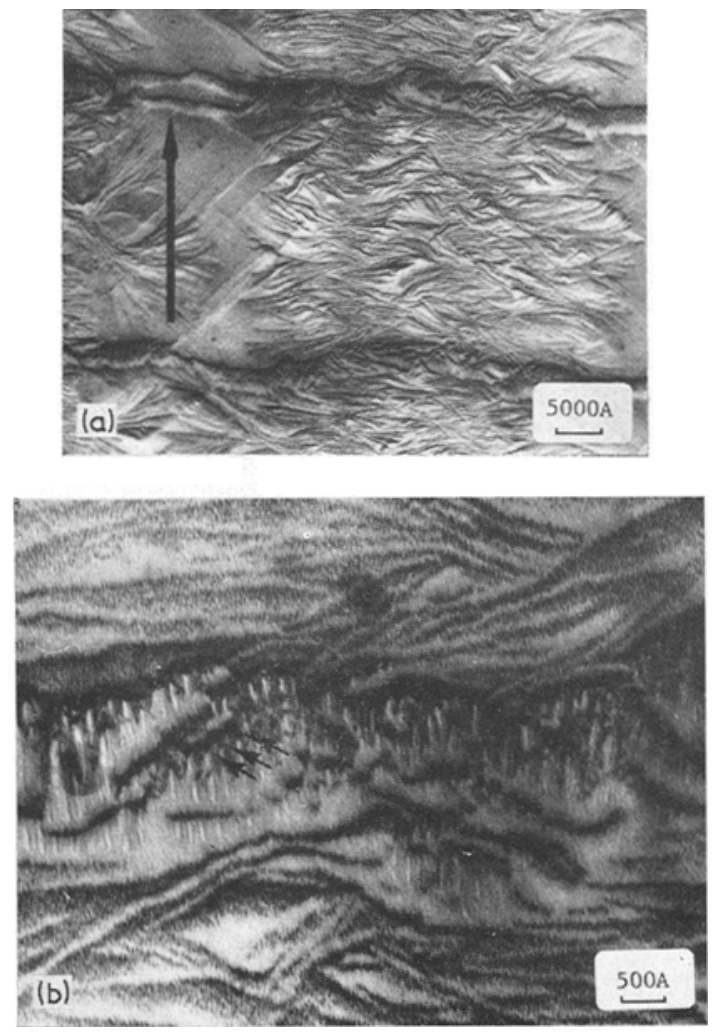

Figure 3 (a) Pt-C surface replica of a semi-crystalline IPS thin film drawn $25 \%$ in a direction perpendicular to the lamellae orientation. The vertical arrow indicates the draw direction. (b) A higher magnification of Fig. 3a. The lamellae break up into $200 \AA$ domains (small arrows) and finally draw down to form fibres 100 to $200 \AA$ in diameter.

indicate several of these 100 to $200 \AA$ domains. It is also shown in Figs. $3 a$ and $b$ that the 100 to $200 \AA$ domains participate in the formation of a 100 to $200 \AA$ fibrillar morphology which is essentially the same craze morphology that we found previously for amorphous atactic polystyrene.

Determining the morphology of the shear bands in thin films was difficult since high magnification micrographs revealed an almost smooth texture with very ill-defined fibrils in the shear orientation direction. To examine the internal structure of these bands more critically, a well-annealed film was uniaxially drawn $25 \%$ to create the craze-shear band structure shown in Fig. Ib. Strips were then cut perpendicular to the original draw direction and redrawn $25 \%$ in that direction. The effect of this second "biaxial" deformation was to "pull apart" the original shear bands, thereby revealing their internal structures. By carefully marking the first and second draw directions before the replication process we were able to establish the draw directions shown in the micrographs of Fig. 4.

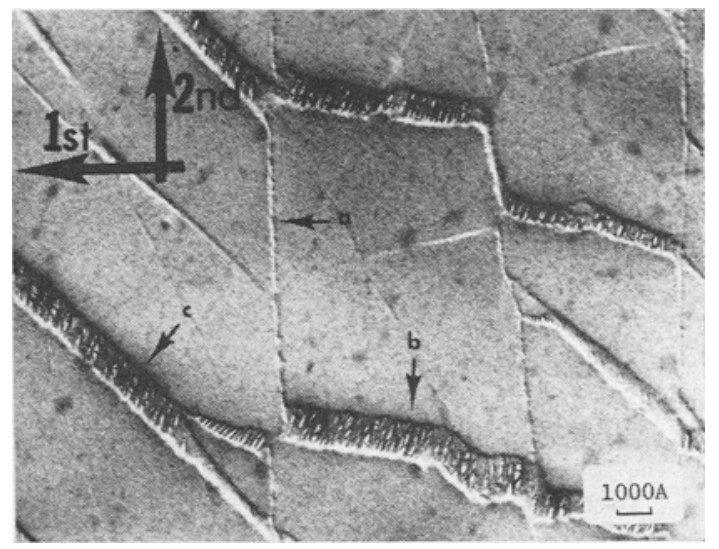

Figure 4 Pt-C surface replica of an APS thin film annealed 3 days at $75^{\circ} \mathrm{C}$, drawn $25 \%$ on a Mylar substrate and then redrawn $25 \%$ in the perpendicular direction. The large arrows indicate the first and second draw directions. Small arrows show a, a collapsed craze formed during the first draw, b, a craze formed during the second draw, and c, a fibrous shear band which opened up during the second draw.

It is shown in Fig. 4 that after the "biaxial" stretch there no longer remain any open crazes perpendicular to the first draw direction. In their place are now linear discontinuities (arrow a in Fig. 4) which we suggest result from the collapse, during the second "biaxial" stretch, of the crazes formed during the first drawing operation. This is reasonable in view of the expected lateral contraction during uniaxial deformation.

It is also evident in Fig. 4 that new "crazes" 
are formed perpendicular to the second draw direction, but in contrast to crazing in a homogeneous unoriented film which always takes place at $90^{\circ}$ to the draw direction, there are, after the "biaxial" draw, two distinct crazing angles at $90^{\circ}$ and $35^{\circ}$ to the second draw direction. The crazes which form at $90^{\circ}$ are indeed crazes which are characteristic of the tensile stretching operation (arrow b in Fig. 4), but those at $35^{\circ}$ lie along the shear direction in the first drawing operation. Both the $90^{\circ}$ and $35^{\circ}$ crazes are fibrous and nearly indistinguishable, except that the fibrils within the $35^{\circ}$ crazes are tilted within the craze (arrow c in Fig. 4), suggesting that the craze is actually a shear band formed during the first stretching process, which has been "pulled apart"revealing a fibrous structure quite equivalent to that of a craze.

\subsection{Bulk samples}

\subsubsection{Shear bands}

Since thin film studies revealed an interesting similarity between the morphology of a shear band and that of a craze, we also examined both

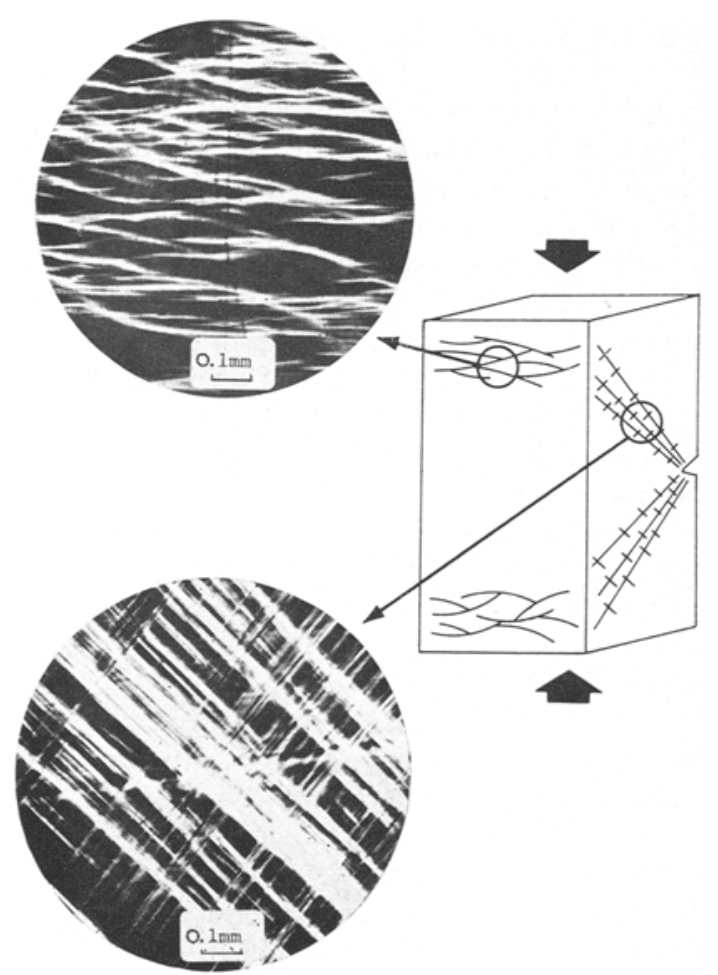

Figure 5 Schematic of an APS compression sample with light micrographs showing shear deformation bands on the side and at the back edge of the sample. shear bands and crazes that were formed in bulk samples deformed in compression.

A schematic of the surface features found in an APS sample deformed in compression is shown in Fig. 5. Light micrographs of thin sections cut both parallel and perpendicular to the notch (an intentionally created stress concentration point) are also shown in Fig. 5. Although the band planes form nearly straight lines as they intersect the side surface of the sample, the band plane itself is clearly not flat through the sample cross-section as can be seen in the upper micrograph of Fig. 5.

Figs. $6 a$ and $b$ are reproduced from our
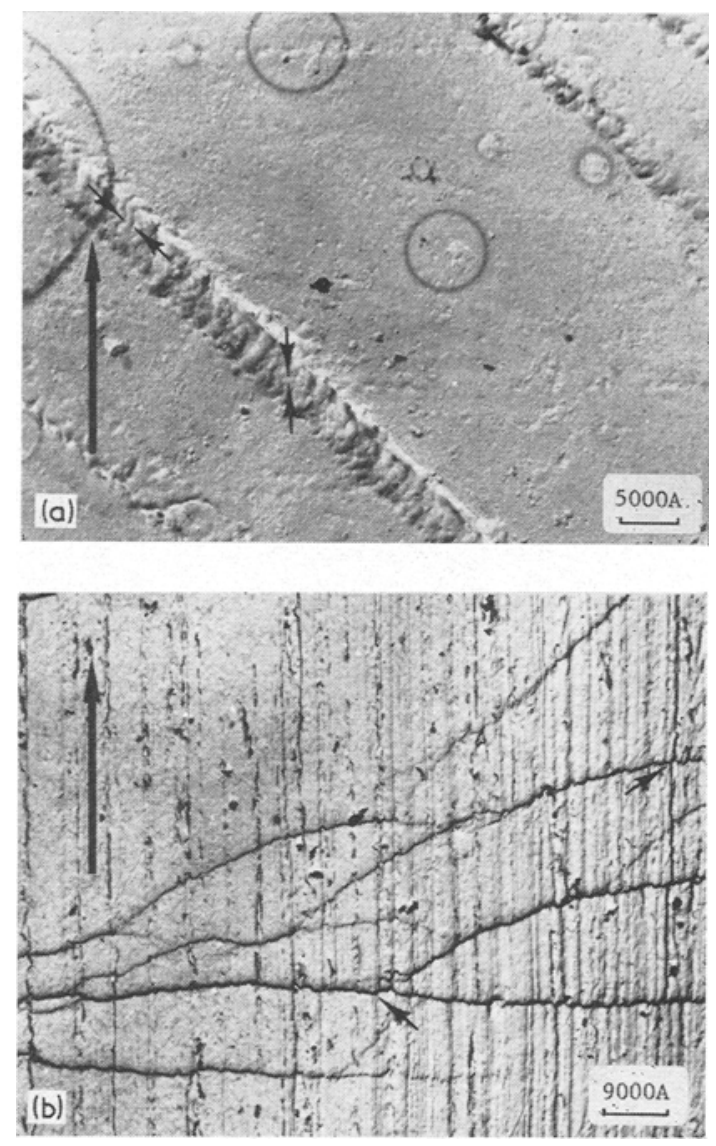

Figure 6 (a) Pt-C surface replica of compression deformation bands produced in APS at $80^{\circ} \mathrm{C}$ and at a nominal strain-rate of $4 \mathrm{~min}^{-1}$. The vertical arrow indicates the compression direction and the small arrows show a 400 to $1000 \AA$ texture within the band [12]. (b) Pt-C surface replica of shear bands as they emerge at the back of the sample. The vertical arrow indicates the compression direction and the small arrows show steps which form as the bands emerge [12]. 
earlier study [13] and show the surface microstructure of a shear band formed at $80^{\circ} \mathrm{C}$ and at a nominal strain rate of $4 \mathrm{~min}^{-1}$. As the bands emerge from the back side of the sample (Fig. $6 \mathrm{~b}$ ), it is again evident that the band plane is neither flat nor continuous across the sample width. The 400 to $1000 \AA$ texture within the band is typical and appears to result from the relative shear displacement of this size structure.

Further evidence for this relative shear displacement was obtained using a different replication technique. First a very thin layer (10 to $20 \AA$ ) of $\mathrm{Pt}$ was evaporated onto the sample and then the sample was deformed. Subsequent replication of the deformed Pt layer produced a surface map of the movement which occurred during deformation. Figs. $7 a$ and $b$ show the
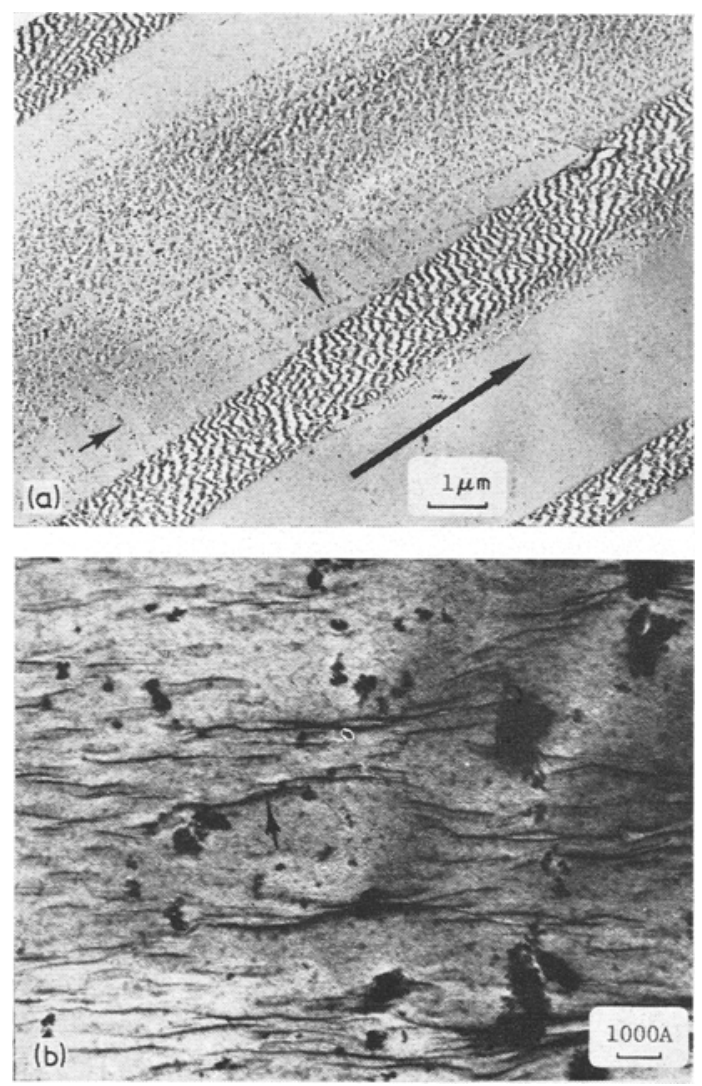

Figure 7 (a) A thin Pt layer was applied to annealed APS before compression. During deformation the $\mathrm{Pt}$ layer breaks up to form 700 to $900 \AA$ ribbons as co-operative shear displacement occurs within the bands. The large arrow indicates the shear direction and the small arrows point to small deformation bands. (b) A thin Pt layer applied to the back side of an APS sample before compression forms ripples as the shear bands emerge. results of this experiment for an APS compression sample, where Fig. 7a shows shear band displacement on the side of the sample and Fig. $7 \mathrm{~b}$ shows the back side of the sample where steps are formed when the band planes emerge. Within the shear bands $(\sim 1 \mu \mathrm{m}$ wide $)$ are 700 to $900 \AA$ Pt ribbons which apparently result from the adjacent operation of several shear processes. The cluster of narrow ( 500 to $900 \AA$ ) bands at the centre of the micrograph (see Fig. 7a) are probably the result of single shear processes. At the back edge of the sample (Fig. 7b) irregular folds are created in the Pt layer as the bands emerge.

In a further effort to reveal the shear band microstructure, several etchants were applied to the surface of the deformed compression samples. Strongly oxidizing acids were used in an attempt to preferentially sever carbon-carbon bonds in the more disordered regions. Artifact produced by the etching method made it difficult to interpret meaningful surface features, but a variation of the usual procedure did produce some interesting results. An APS sample was polished and then annealed above $T_{\mathrm{g}}$ to produce a smooth surface. Chromic-sulphuric acid (50:50) was applied full strength to the surface for $2 \mathrm{~h}$ at room temperature. The acid was then washed off in distilled water and the sample was deformed in compression. Surface replicas shown in Figs. $8 \mathrm{a}$ and $\mathrm{b}$ contrast shear bands in a sample that was not etched before deformation with those formed in an etched sample. Even with no etch the band appears fibrous with a lightly defined 200 to $500 \AA$ texture along the fibre axis (Fig. 8a). On the surface of the etched sample, however, the texture is much better defined, suggesting that the acid may indeed have a preferential etching effect between the 200 to $500 \AA$ domains.

A novel technique was developed to examine the internal morphology of an APS compression shear band. A sample was cut perpendicular to the compression direction at a point half way between the notch and the sample end (see Fig. 9). Both of the internal compression surfaces were then polished and both halves of the sample were annealed. The internal "free surfaces" were then placed together again and the sample was compressed. Since the test was performed in compression, the two surfaces were always in intimate contact which allowed the plastic deformation zone (shear bands) to propagate across the interface without difficulty. After compression the two surfaces were separated, 

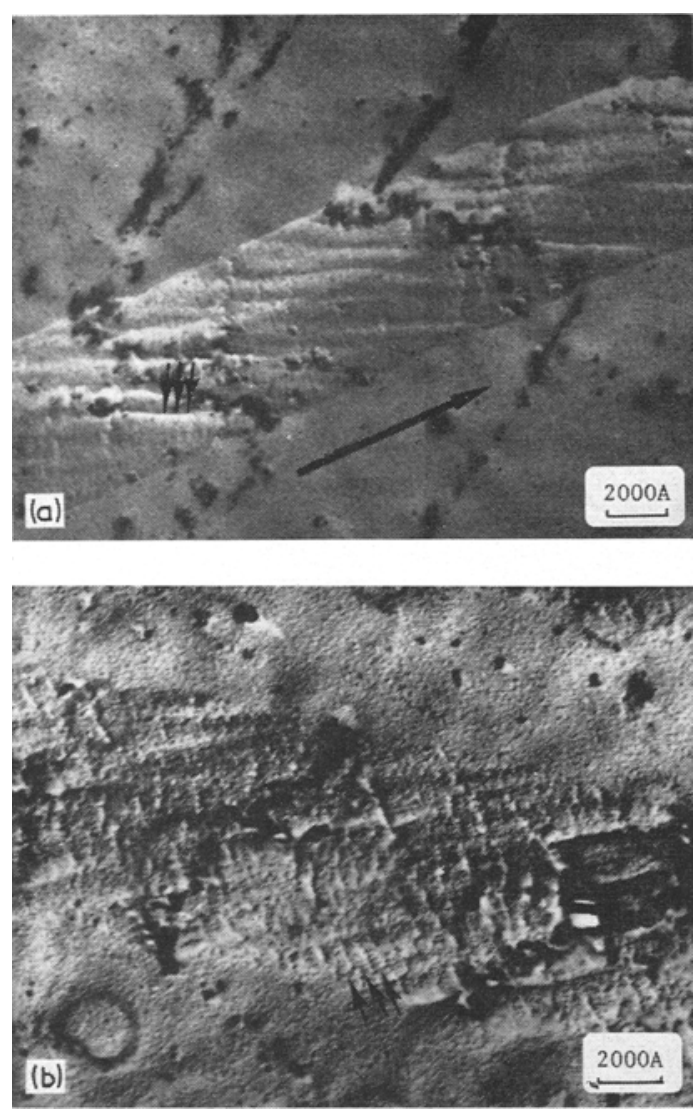

Figure 8 (a) Pt-C surface replica of an APS sample polished with fine $(0.05 \mu \mathrm{m}) \mathrm{Al}_{2} \mathrm{O}_{3}$, annealed 3 days at $75^{\circ} \mathrm{C}$ and compressed. The dark lines of residual $\mathrm{Al}_{2} \mathrm{O}_{3}$ jog across the shear band. The large arrow indicates the shear direction and the small arrows show a 200 to $500 \AA$ texture along the fibres. (b) If the sample is etched $2 \mathrm{~h}$ in chromic-sulphuric acid before compression the texture along the fibres becomes highly emphasized.

revealing what was considered to be a good approximation of the internal microstructure (Figs. 10a and b). While recognizing the possible influence of the polished surfaces as well as the possibility of slip between these surfaces, we examined this internal structure and compared it with the morphology that we found on the external surfaces (see, for example, Figs. $6 a$ and b).

These internal compression surfaces were $\mathrm{Pt}$ shadowed at an angle of $30^{\circ}$, but in two directions: perpendicular (Fig. 10a) and parallel (Fig. 10b) to the planar orientation of the shear bands at the interface. Fig. 10a shows the results of the perpendicular shadow where well-defined

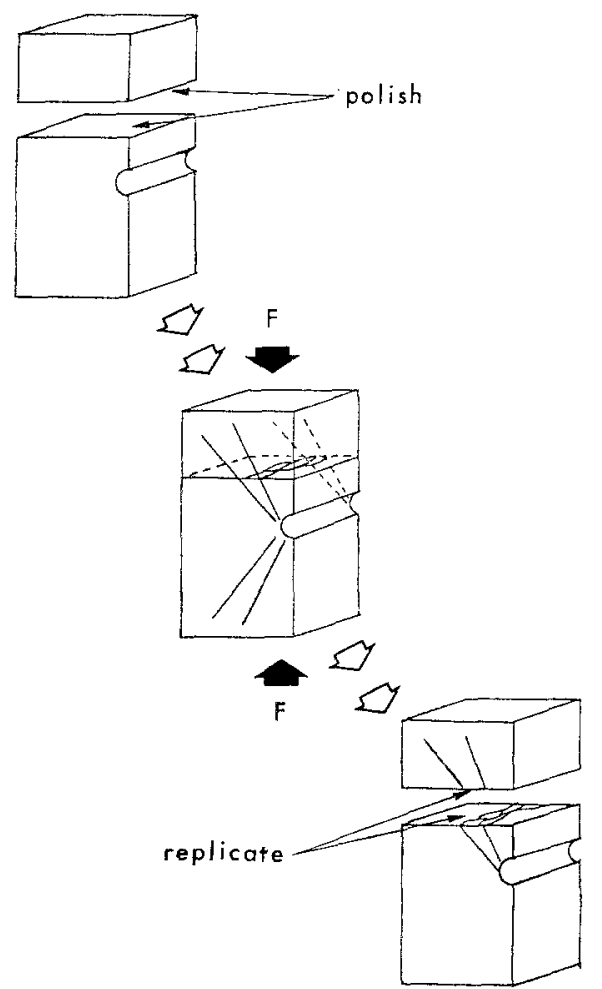

Figure 9 A method for producing an "internal" compression surface.

steps can be seen. The parallel shadowing in Fig. 10b elucidates a 200 to $500 \AA$ fibrous morphology within the shear bands.

Since localized deformation is a function of both temperature and strain rate, shear band morphology was also studied as a function of these variables. Although the band widths varied from several hundred Angstroms to several microns, width was found not to be a consistent function of either temperature or strain rate.

\subsection{Bulk samples}

\subsubsection{Crazes}

When a notched polystyrene sample is compressed, packets of shear bands initiate at the notch and propagate through the sample [13, 16]. As the deformation proceeds and the sample becomes increasingly "bowed", tensile stresses are induced in the sample surface. Because the deformation is permanent, these surface stresses remain even after the compressive load is removed. Upon ageing for 2 to 6 weeks at room temperature these surface stresses cause crazes to form in the polysty'rene samples which can then 

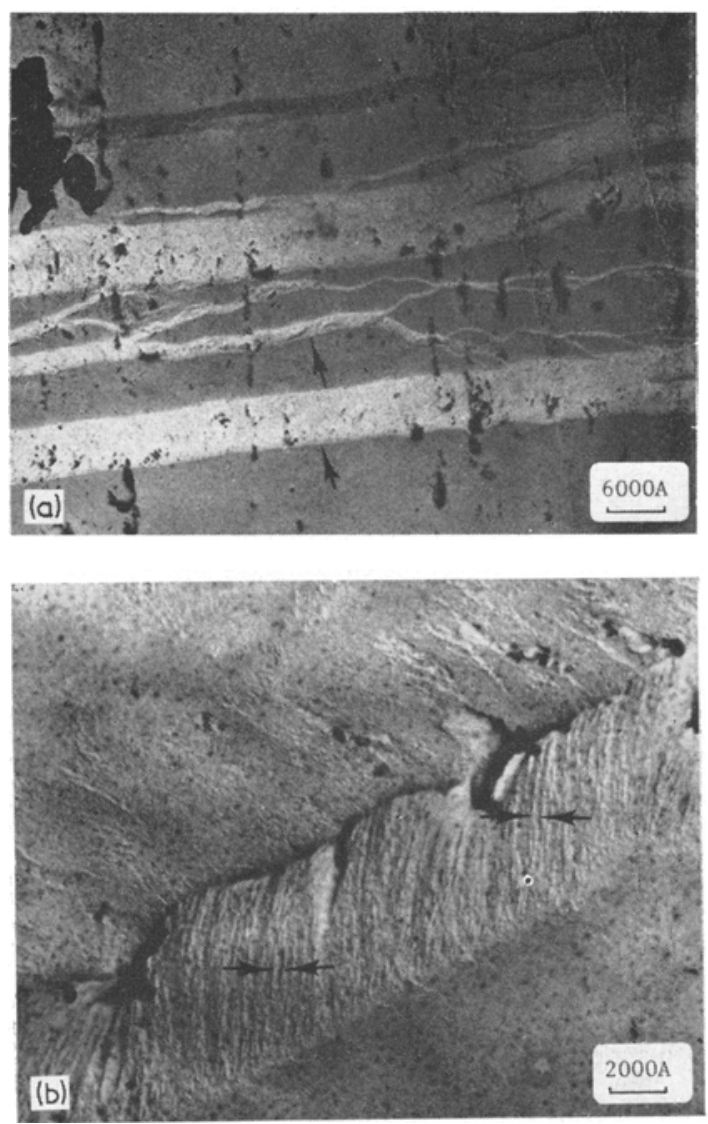

Figure 10 (a) Pt-C surface replica of an APS internal compression surface. The shadow was applied perpendicular to the band planes thereby revealing steps (small arrows). The compression direction is into the plane of the paper. (b) Pt-C surface replica of an internal APS compression surface. With the shadow parallel to the band plane, 100 to $500 \AA$ fibres are revealed within the band.

be observed using conventional replication techniques and the electron microscope.

Figs. $11 \mathrm{a}$ and $\mathrm{b}$ show that the morphology at the edge of such a craze is less fibrous than is the craze centre. Deformation at the edge appears to proceed by a nonhomogeneous slip mechanism, leaving relatively well-defined 200 to $700 \AA$ domains. As deformation continues and orientation increases in the craze centre, 200 to $700 \AA$ fibres form from these domains.

\subsection{Effects of molecular weight}

Compression samples were moulded from several different narrow distribution molecular weight atactic polystyrenes having weight average molecular weights of $402000,265000,113000$,
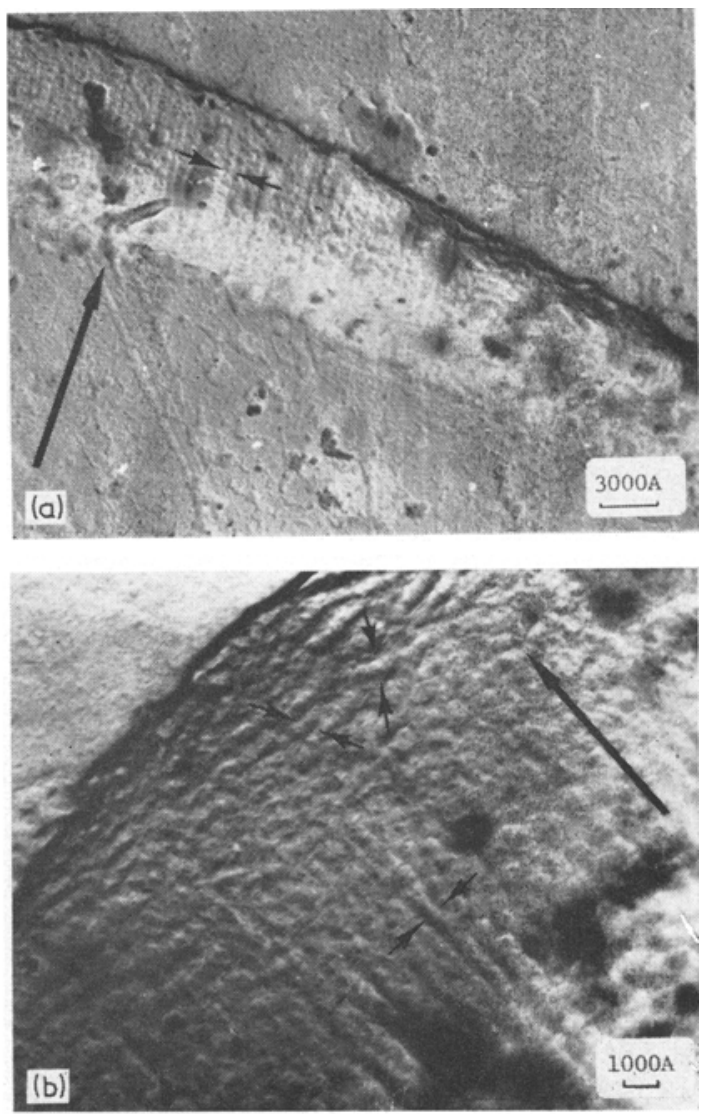

Figure 11 (a) Pt-C surface replica of a craze formed in an APS compression sample, where the large arrow indicates the direction of the surface tensile stresses. Small arrows show fibres within the craze. (b) A higher magnification of the craze in Fig. 11a reveals 200 to $700 \AA$ domains at the craze edge and 200 to $700 \AA$ fibres in the highly oriented craze centre (small arrows).

and 15000. Regardless of thermal history, shear deformation bands could not be formed in the low molecular weight (15000) polymer; fracture always intervened, even at strain-rates as low as $0.004 \mathrm{~min}^{-1}$. All of the other higher molecular weight samples, however, exhibited deformed morphologies that were indistinguishable from. that of the general purpose polystyrene, GPAPS, after compression, In each case compression resulted in localized shear band formation and surface replicas revealed a band microstructure identical to that previously described for general purpose polystyrene. Internal compression surfaces obtained in the same way as was described previously (see Fig. 9) also revealed fibrous shear bands with fibres again 
several hundred Angstroms in diameter and almost indistinguishable from those shown in Figs. 10a and $\mathrm{b}$ for general purpose polystyrene.

To test the effects of molecular weight on thin film morphology, and also to test a wider range of low molecular weights, thin films were cast from several narrow distribution molecular weight atactic polystyrenes with weight average molecular weights of $411000,51000,19800$, and 4800. These films were drawn $25 \%$ on Mylar substrates and their deformed morphologies compared. In each case shear bands formed during deformation, even in the case of the very low molecular weight (4800) polymer. The bands did, however, become increasingly less well defined with decreasing molecular weight (see Figs. 12 to

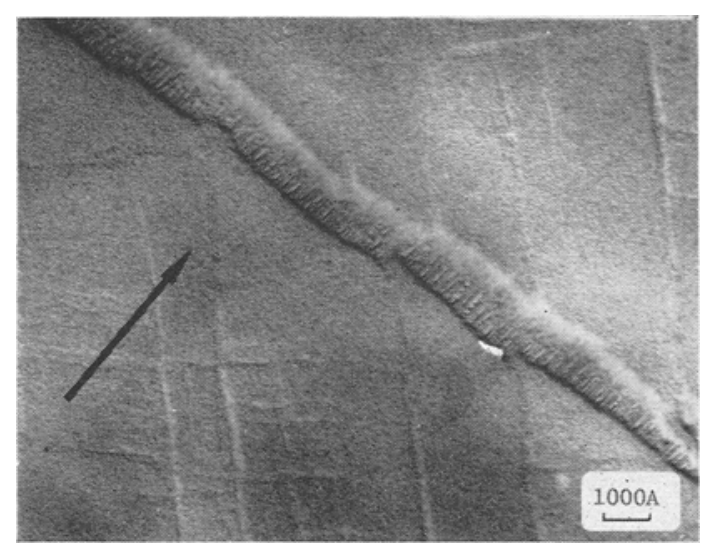

Figure $12 \mathrm{Pt}-\mathrm{C}$ surface replica of an APS thin film (mol wt 402000) drawn $25 \%$ on a Mylar substrate. The large arrow indicates the draw direction.

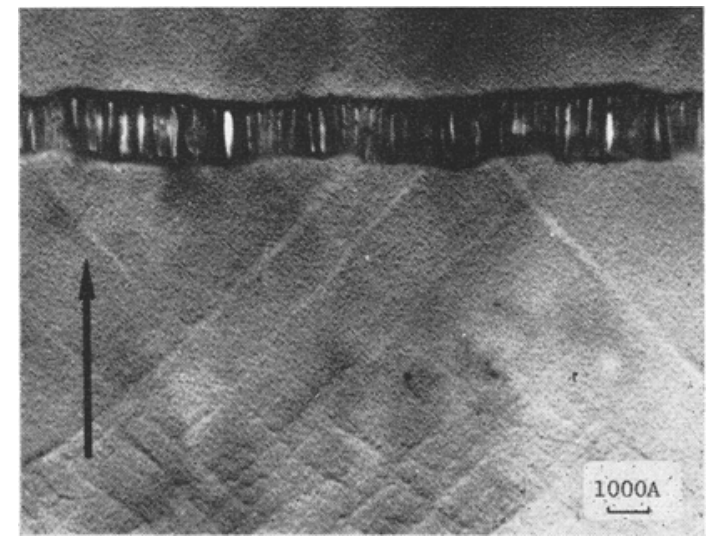

Figure $13 \mathrm{Pt}$-C surface replica of an APS thin film (mol wt 51000 ) drawn $25 \%$ on a Mylar substrate. The vertical arrow indicates the draw direction.

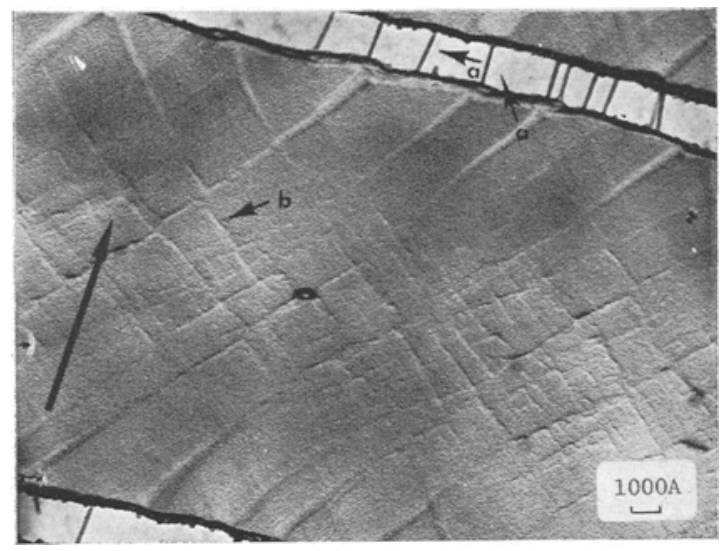

Figure 14 Pt-C surface replica of an APS thin film (mol wt 19800) drawn $25 \%$ on a Mylar substrate. The large arrow indicates the draw direction and the small arrows show a, a craze where few fibres span the opening, and $b$, shear lines that are becoming less distinct with decreasing molecular weight.

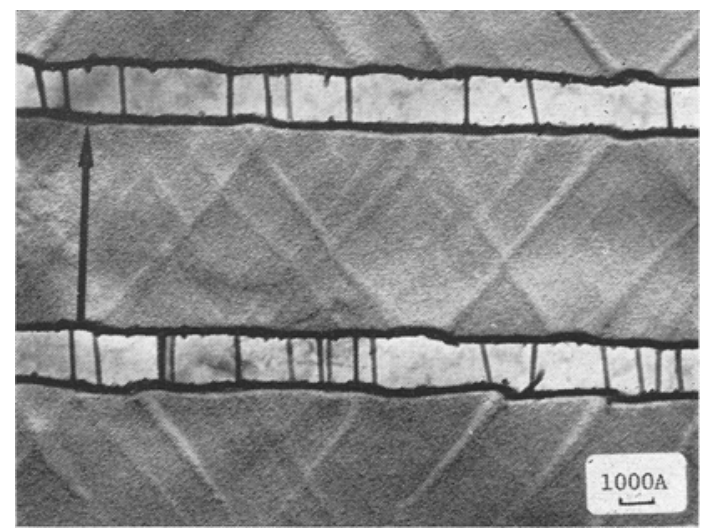

Figure $15 \mathrm{Pt}-\mathrm{C}$ surface replica of an APS thin film (mol wt 4800) drawn $25 \%$ on a Mylar substrate. The shear lines have become even less distinct in this very low molecular weight film.

15). These micrographs also show that the continuity of the fibrous crazed regions decreases with decreasing molecular weight. In the very low molecular weight polymers (Figs. 14 and 15) very few fibres are able to span the craze width. Sequential stretching experiments performed on these same thin films also revealed that fibres in the low molecular weight polymers were unable to span the crazes (see Figs. 16 and 17). However, it is also noted in these micrographs that any fibres which do span the craze are always on the 


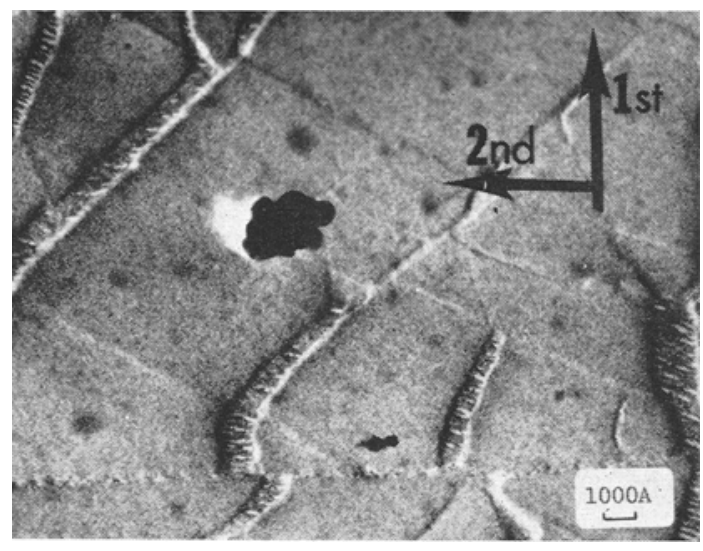

Figure $16 \mathrm{Pt}-\mathrm{C}$ surface replica of an APS thin film (mol wt 402000 ) annealed 6 days at $75^{\circ} \mathrm{C}$, drawn $25 \%$ on Mylar and then redrawn $25 \%$ in the cross direction. The arrows show the first and second draw directions.

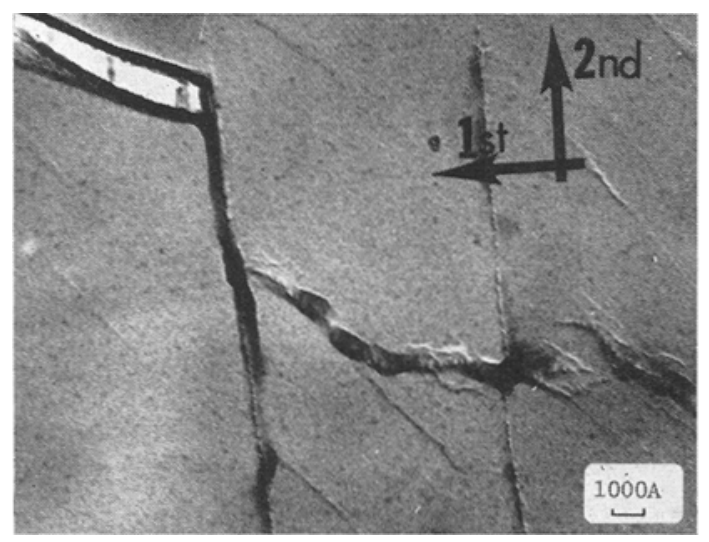

Figure $17 \mathrm{Pt}-\mathrm{C}$ surface replica of an APS thin film (mol wt $=19800$ ) annealed 6 days at $75^{\circ} \mathrm{C}$, drawn $25 \%$ on Mylar and then redrawn $25 \%$ in the cross direction. The arrows show the first and second draw directions.

order of several hundred Angstroms in diameter regardless of molecular weight.

\section{Discussion}

This investigation of craze and shear band morphology was limited to the examination of surface replicas in the electron microscope and hence we were not able to resolve any significant change in the polystyrene fine structure $(<50 \AA)$ which Yeh identified in his studies. We did, however, resolve distinct microstructural features which varied from 50 to $1000 \AA$ in size and appeared to influence the localized deformation processes in polystyrene. In this regard our results are in agreement with the study by Beahan et al in which polystyrene craze fibres were shown to consist of 75 to $150 \AA$ domains. In our study the sizes of the fibrils and domains vary between 50 and $1000 \AA$, but there seems to be a reasonable explanation for this variation which is consistent with previous studies. Kambour and Holick [8] showed that craze morphology can vary from a nonfibrillated structure at the craze edge to a highly oriented fibrillar structure at the craze centre. A subsequent study by Beahan et al showed that 200 to $400 \AA$ fibrils which appear initially within a craze decrease to a more regular diameter of $250 \AA$ as the craze widens. In fully developed crazes these fibrils finally approach a limiting width of 75 to $150 \AA$, suggesting that fibril diameter and domain size do correlate with degree of orientation. We suggest that the size variation of the microstructural features seen in this study also reflects varying degrees of orientation and that "necking down" of the craze and shear band fibrils is dictated by a domain size which is characteristic of the polymer microstructure. We find, for example, that microstructural features within the bands vary from 200 to $1000 \AA$ in bulk samples, but are generally greater than $400 \AA$. Within the more highly oriented thin films crazes, however, the fibril diameters average 200 to $300 \AA$ and interconnecting links as narrow as $50 \AA$ can be identified (see Fig. 1a). These $50 \AA$ interconnecting links were also identified by Beahan et al which suggests that further necking down of the 200 to $300 \AA$ fibrils might occur within the APS crazes, but that the integrity of the $30 \AA$ fine structure [12] is great enough to prevent this from occurring to any great extent under conditions which normally initiate crazing and plastic yield.

It is interesting to contrast our experiments with semicrystalline isotactic polystyrene to the results of Yeh and Geil [24] who observed a very similar 200 to $500 \AA$ beaded fibrous structure within the drawn regions of crystalline polyethylene spherulites suggesting that deformation of crystalline and non-crystalline polymers might be quite analogous and that the deformation mechanisms in both may indeed depend on the influence of microstructural domains.

This study further suggests that the morphologies of "crazes" and "shear bands" in atactic polystyrene, as well as in both amorphous and semi-crystalline isotactic polystyrene, are quite 
similar. Both modes of plastic deformation depend upon the relative displacement of 100 to $1000 \AA$ domains. As deformation continues and orientation within the deformation zoneincreases, fibres which vary from 50 to $700 \AA$ are formed. However, since crazing is controlled by a tensile stress [1], the fibrous matrix contains voids which result from the restricted lateral Poisson contraction. Shear banding, on the other hand, is controlled by a shear stress which actually encourages lateral movement and therefore prevents void formation. Although our observations of craze morphology are consistent with those of other investigators [1-3], there are no comparable detailed morphological studies of shear banding which suggest the near equivalence between the two that we find in this study.

Although void formation within a craze is the consequence of a tensile stress, our micrographs (Figs. 11a and b) suggest that the crazing phenomenon may not occur completely independent from the shear field within a stressed sample. In agreement with the previous study by Kambour and Holick [8], it is shown in Fig. 11 b that craze morphology does vary from a nonfibrillated structure at the craze edge to a highly fibrillated structure at the craze centre. At the centre the fibres are definitely aligned along the tensile direction, but there is no definite tensile orientation of the 200 to $700 \AA$ domains at the craze edge, suggesting that their relative displacement might be caused by shear. In fact, on the average, the 200 to $700 \AA$ texture at the craze edge (Fig. 11b) is quite similar in both size and relative shear orientation to the 300 to $450 \AA$ texture which we [13] observed in uniformly cold drawn APS where no crazing was evident.

It is also significant that shear band width is not a unique function of either temperature or strain-rate suggesting that a shear band is simply a zone of localized deformation which results from the combined operation of several individual shear processes. The band itself, therefore, is probably not uniquely characteristic of the polymer microstructure, whereas the local displacement which occurs within a band is very likely a consequence of the individual shear processes and, therefore, is uniquely related to the undeformed polymer morphology.

Based on this study, we can also offer an explanation for the observation of Harris and Ward [6] who noted that when oriented polyethylene terephthalate is redrawn, crazes form both parallel to the initial draw direction (tensile crazes) and in a direction which is almost parallel to the deformation bands observed at yield (shear crazes). When an oriented film is redrawn, the resolved stress component which is perpendicular to the original shear direction will tend to "open up" shear bands that lie along this direction, as was the case in our oriented polystyrene films. Since shear band and craze morphologies are very similar, and open shear band looks very much like a craze and could be what Harris and Ward called a "shear craze". Of course whether this deformation zone is defined to be a craze or a shear band at this point is largely a semantic issue.

Finally, our study suggests that neither the deformed morphology nor the deformation mechanism depend to a great extent on molecular weight. Plastic deformation proceeds by localized shear band formation even in the low molecular weight polystyrenes. Fibres which develop within the shear bands are always on the order of several hundred Angstroms in diameter regardless of molecular weight, suggesting that the basic unit of movement is probably not highly dependent upon molecular weight. The increasingly diffuse shear bands and the inability for fibres to span wide openings in the low molecular weight polymers (i.e. $<20000$ ), however, imply that the number of tie molecules between structural units is highly dependent upon molecular weight, at least for molecular weights $<20000$. Rudd [20] and Kambour [21] have both reported that craze strength and time to failure also decrease with decreasing molecular weight, and they explain their results in terms of decreased numbers of tie molecules. However, both studies concerned themselves with relatively high molecular weight polymers $(>90000)$. In comparison to our work it is interesting to note that for molecular weights 20000 there is not sufficient polymer integrity for either shear bands or crazes to fully develop suggesting that below the critical molecular weight there are so few tie molecules between domains that the polymer fails before large scale plastic deformation can occur.

\section{References}

1. S. RABINOWITZ and P. BEARDMORE, CRC Critical Reviews in Macromolecular Science (January, 1972) p. 13

2. R. P. KAMBOUR and R. E. ROBERTSON, The Mechanical Properties of Plastics, in "Materials Science of Polymers" (North Holland, Amsterdam, 1971). 
3. R. P. камвour, "Appl. Polymer Symp.", no. 7 (Interscience, New York, 1968) p. 215.

4. P. Beahan, M. Bevis, and D. Hull, Phil. Mag. 24 (1971) 192.

5. R. P. KAMBoUr and R. R. RUSSELl, Polymer 12 (1971) 237.

6. J. S. HARRIS and I. M. WARD, J. Mater. Sci. 5 (1970) 573.

7. J. MURRAY and D. HULL, Polymer Letts. 8 (1970) 159.

8. R. P. KAMBOUR and A. S. HOLICK, J. Polymer Sci. Pt $A-2,7$ (1969) 1393.

9. Idem, ACS Polymer Preprints 10 (1969) 1182.

10. R. P. KAMBoUR, Polymer 5 (1964) 143.

11. o. K. SPURR and W.D. Niegisch, $J$. Appl. Polymer Sci. 6 (1962) 585.

12. G. S. Y. YEH, J. Macromol. Sci. (Phys.) B6(3) (1972). 451.
13. T. E. BRADY and G. S. Y. YEH, I. Appl. Phys. 42 (1971) 4622.

14. P. B. BOWDEN and s. RAHA, Phil. Mag. 22 (1970) 463.

15. R, e. robertson, $J$. Polymer Sci, Pt $A-27$ (1969) 1315.

16. A, S. ARGON, R. D. ANDREWS, J. A. GODRICK, and W. Whit ney, J. Appl. Phys. 39 (1968) 1899.

17. W. WHITNEY, ibid 34 (1963) 3633.

18. D. A. ZAUKELIES, ibid 33 (1962) 2797.

19. R. N. HAWARD, B. M. MURPHY, and E. F. T. White, J. Polymer Sci. A-2, 9 (1971) 801.

20. J. F. RUDD, Polymer Letts. I (1963) 1.

21. R, P. Kambour, Polymer Eng. Sci. 8 (1968) 281.

22. E. BAER, private communication.

23. D. HULL, private communication.

24. G. S. Y. YEH and P. H. GEIL, J. Macromol. Sci. (Phys.) B2(1), (1968) 29.

Received 12 October 1972 and accepted 12 February 1973 